\title{
Detonation Tube Impulse in Subatmospheric Environments
}

\author{
M. Cooper* \\ Sandia National Laboratories, Albuquerque, New Mexico 87185-0834 \\ and \\ J. E. Shepherd ${ }^{\dagger}$ \\ California Institute of Technology, Pasadena, California 91125
}

\begin{abstract}
Thrust from a multicycle pulse detonation engine operating at practical flight altitudes will vary with surrounding environment pressure. We have carried out the first experimental study using a detonation tube hung in a ballistic pendulum arrangement within a large pressure vessel to determine the effect that the environment has on the single-cycle impulse. Air pressure decreased below $100 \mathrm{kPa}$, whereas initial pressure of the stoichiometric ethyleneoxygen mixture inside the tube varied between 100 and $30 \mathrm{kPa}$. The original impulse model (Wintenberger et al., Journal of Propulsion and Power, Vol. 19, No. 1, 2002, pp. 22-38) was modified to predict the observed increase in impulse and blowdown time as the environmental pressure decreased below $1 \mathrm{~atm}$. Comparisons between the impulse from detonation tubes and ideal steady-flow rockets indicate incomplete expansion of the detonation tube exhaust, resulting in a $37 \%$ difference in impulse at a pressure ratio (ratio of pressure behind the Taylor wave to environmental pressure) of 100 .
\end{abstract}

\section{Nomenclature}

$A=$ area

$c \quad=$ speed of sound

$F=$ force

$F_{D}=$ force due to diaphragm

$g=$ gravitational acceleration

$h=$ enthalpy per unit mass

$I \quad=$ impulse

$I_{\mathrm{SP}}=$ mixture-based specific impulse

$I_{V}=$ impulse normalized by the tube volume

$K=$ model proportionality constant

$L=$ tube length

$L_{p}=$ pendulum arm length

$M_{p}=$ pendulum mass

$P=$ pressure

$q=$ effective energy release per unit mass of mixture

$R=$ perfect gas constant

$T=$ temperature

$t=$ time

$t_{1}=$ time taken by the detonation wave to reach the open tube end

$t_{2}=$ time taken by the first reflected characteristic to reach the thrust surface

$t_{3}=$ time associated with pressure decay period

$U_{\mathrm{CJ}}=$ Chapman-Jouguet detonation velocity

$V=$ tube volume

$\alpha=$ nondimensional parameter corresponding to time $t_{2}$

$\beta=$ nondimensional parameter corresponding to pressure decay period

$\gamma=$ specific heat ratio

$\Delta x=$ maximum horizontal displacement

$\Pi=$ nondimensional pressure, $\left[P(t)-P_{0}\right] /\left[P_{3}-P_{0}\right]$

$\rho=$ density

Received 5 April 2005; revision received 27 September 2005; accepted for publication 27 September 2005. Copyright (C) 2005 by California Institute of Technology. Published by the American Institute of Aeronautics and Astronautics, Inc., with permission. Copies of this paper may be made for personal or internal use, on condition that the copier pay the $\$ 10.00$ per-copy fee to the Copyright Clearance Center, Inc., 222 Rosewood Drive, Danvers, MA 01923; include the code 0748-4658/06 \$10.00 in correspondence with the CCC.

*Postdoctoral Appointee, Thermal, Fluid and Aero Experimental Sciences Department. Member AIAA.

†Professor, Aeronautics Department. Member AIAA.
Subscripts

$t=$ stagnation properties

$0=$ state of environment

$1=$ reactant state

2 = Chapman-Jouguet detonation state

$3=$ state in stagnant flow region behind the Taylor wave

\section{Introduction}

$\mathbf{M}$ OTIVATION for this study comes from the continued development of a novel propulsive device called a pulse detonation engine (PDE) that is based on intermittent detonation to generate quasi-steady thrust. Multicycle performance estimates ${ }^{1,2}$ for fully filled air-breathing PDEs without exit nozzles currently exist for varying flight Mach numbers. These results suggest inefficient operation due to incomplete expansion of the exit flow, yet no experimental data exist with which to validate these estimates.

For PDE performance to be comparable to that of existing propulsion systems, it has been proposed to use some type of exit nozzle. Known from the analysis of steady flow nozzles, the nozzle pressure ratio determines the nozzle effectiveness and depends directly on the environmental pressure, which varies as a function of altitude. Operation at higher altitude increases the nozzle pressure ratio, enabling more thermal energy of the exhaust products to be converted into kinetic energy, thus increasing the thrust transferred to the engine. Before the effect of nozzles on detonation tubes can be quantified, the effect that the environmental pressure has on the impulse from fully filled straight detonation tubes must be understood. The environmental temperature also varies with altitude, but this effect on impulse is beyond the scope of this work. The data presented here provide a baseline from which detonation tube nozzles can be evaluated and enable the effect of increased blowdown time due to exhausting into lower pressures to be separated from the additional flow expansion provided by a nozzle. Comparisons of the detonation tube impulses to estimates assuming ideal steady flow provide a measure of detonation exhaust under expansion. Although such a comparison is not strictly valid, because the flow exhaust from a detonation tube is unsteady and not pressure-matched to the environment, the observed differences suggest the magnitude of impulse that could possibly be gained by adding a perfectly designed nozzle.

Historically, single-cycle ballistic pendulum experiments have been instrumental in quantifying the maximum impulses obtained for specific operating conditions which, until now, have only investigated in-tube parameters such as the initial pressure, equivalence ratio, and diluent of the explosive mixture, internal obstacle configurations, and ignition sources. For this reason, we utilize a ballistic 
pendulum arrangement in conducting the first systematic experimental investigation of detonation tube impulse as a function of environment pressure. A simplified detonation tube, consisting of a cylinder closed at one end and open at the other, is used. The existing impulse model $^{3}$ is extended to include the effect of environment pressure.

\section{Impulse Model for $\boldsymbol{P}_{\mathbf{0}} \neq \boldsymbol{P}_{\mathbf{1}}$}

A detonation tube is best analyzed with a control volume ${ }^{3}$ that surrounds the tube walls (Fig. 1). The idealized thrust surface pressure history for the situation when $P_{0} \neq P_{1}$ is illustrated in Fig. 2. The impulse is predicted by integrating the forces acting on the control volume of Fig. 1,

$$
I=\int_{0}^{\infty} \sum F \mathrm{~d} t=I_{1}+I_{2}+I_{3}
$$

and consists of contributions from the three time periods illustrated in Fig. 2.

During the time period $t_{1}=L / U_{\mathrm{CJ}}$, the thrust surface experiences a force from the pressure differential $P_{3}-P_{0}$, whereas the open tube end experiences a constant force from the pressure differential $P_{0}-P_{1}$. This pressure difference at the open end is supported by the diaphragm that passes through the control surface (Fig. 1), generating a force

$$
F_{D}=\int_{0}^{t_{1}}\left(P_{0}-P_{1}\right) \cdot A \mathrm{~d} t
$$

not considered in previous impulse models. ${ }^{3,4}$ The impulse integral during time $t_{1}$ is

$$
\begin{aligned}
I_{1} & =\int_{0}^{t_{1}}\left(P_{3}-P_{0}\right) \cdot A \mathrm{~d} t+F_{D} \\
& =\int_{0}^{t_{1}}\left(P_{3}-P_{1}\right) \cdot A \mathrm{~d} t=\left(P_{3}-P_{1}\right) A t_{1} \\
& =\frac{\left(P_{3}-P_{1}\right) V}{U_{\mathrm{CJ}}}
\end{aligned}
$$

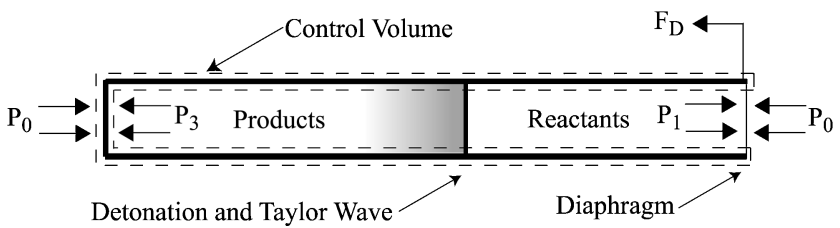

Fig. 1 Detonation tube control volume when the initial combustible mixture is sealed inside the tube with a diaphragm and the detonation wave has not reached the open end.

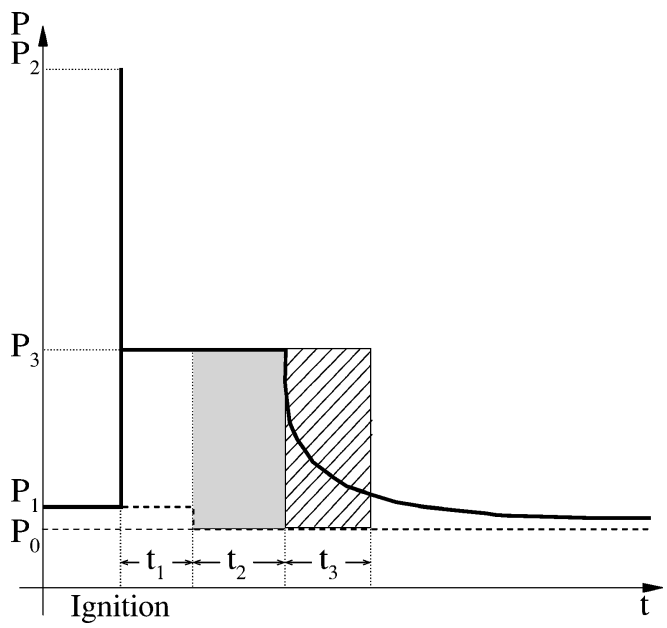

Fig. 2 Idealized thrust surface pressure history for tubes with $P_{1}$ not equal to $P_{0}$.
Time $t_{2}$ is required for the reflected wave from the open tube end to reach the thrust surface. During this time, the thrust surface pressure history can be integrated directly from the pressure difference across the thrust surface and is scaled ${ }^{3}$ with the nondimensional parameter $\alpha=t_{2} c_{3} / L$ :

$$
\begin{aligned}
I_{2} & =\int_{t_{1}}^{t_{1}+t_{2}}\left(P_{3}-P_{0}\right) \cdot A \mathrm{~d} t=\left(P_{3}-P_{0}\right) A t_{2} \\
& =\frac{\left(P_{3}-P_{0}\right) \alpha V}{c_{3}}
\end{aligned}
$$

During time $t_{3}$, the rate of pressure decay at the thrust surface is determined by the environmental pressure and the relative sound speeds in the gases. The impulse $I_{3}$ during this time is scaled ${ }^{3}$ with the nondimensional parameter:

$$
\begin{gathered}
\beta=\int_{\tau_{1}+\tau_{2}}^{\infty} \Pi(\tau) \mathrm{d} \tau \\
I_{3}=\int_{t_{1}+t_{2}}^{\infty}\left[P(t)-P_{0}\right] \cdot A \mathrm{~d} t=\frac{\left(P_{3}-P_{0}\right) V}{c_{3}} \int_{\tau_{1}+\tau_{2}}^{\infty} \Pi(\tau) \mathrm{d} \tau \\
=\frac{\left(P_{3}-P_{0}\right) \beta V}{c_{3}}
\end{gathered}
$$

With the value of $\beta$, a characteristic time $t_{3}=\beta L / c_{3}$ is defined ${ }^{3}$ that represents the hatched region in Fig. 2.

When $P_{0}=P_{1}$, the pressure decay integral was assumed ${ }^{3}$ to have a constant value of $\beta=0.53$. Decreasing the environment pressure will increase the blowdown time $t_{3}$ along with the corresponding value of $\beta$. This increase in blowdown time is evident from the measured thrust surface pressure histories, shown in Fig. 3, for environmental pressures of $100 \mathrm{kPa}$ and $1.4 \mathrm{kPa}$. Because the exhaust is choked throughout most of the process, the pressures are identical until the value of $P_{0}$ is nearly reached. The traces clearly show that additional time is required for the detonation tube to equilibrate to the lower environmental pressures (approximately $5 \mathrm{~ms}$ for $P_{0}=100 \mathrm{kPa}$ if the underpressure region is not considered and approximately $7.5 \mathrm{~ms}$ for $P_{0}=1.4 \mathrm{kPa}$ ). Thus, simply decreasing the environmental pressure by almost $100 \%$ causes a $50 \%$ increase in the blowdown time. Although this increase in blowdown time positively affects the single-cycle impulse, it should be noted that the thrust of multicycle PDEs may be negatively affected if high cycle frequencies are required.

The three components of the impulse for times $t_{1}, t_{2}$, and $t_{3}$ are summed to yield the total specific impulse:

$$
I_{\mathrm{SP}}=I / V \rho_{1} g=K\left(P_{3}-P_{0}\right) / \rho_{1} g U_{\mathrm{CJ}}
$$

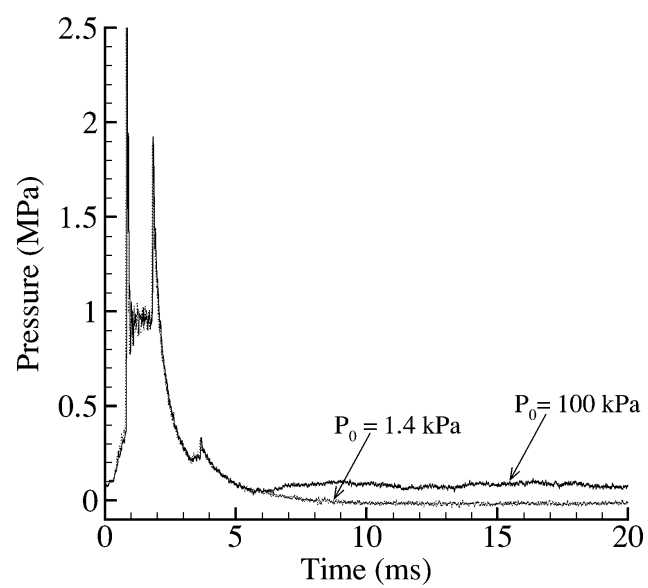

Fig. 3 Measured thrust surface pressure histories with $P_{1}=80 \mathrm{kPa}$ and $P_{0}=100$ and $1.4 \mathrm{kPa}$. 
This relationship for the impulse equals that previously determined by Wintenberger et al., ${ }^{3}$ except that now we find that $K$ depends not only on the energy content $q / R T_{1}$ and the specific heat ratio $\gamma$ as was determined previously, ${ }^{3}$ but also on the environmental pressure $P_{0} / P_{1}$

$$
\begin{aligned}
K= & K\left(\gamma, q / R T_{1}, P_{0} / P_{1}\right) \\
& =\left[\frac{\left(P_{3} / P_{1}-1\right)}{\left(P_{3} / P_{1}-P_{0} / P_{1}\right)}+\alpha \frac{U_{\mathrm{CJ}}}{c_{3}}+\beta \frac{U_{\mathrm{CJ}}}{c_{3}}\right]
\end{aligned}
$$

Equation (6) differs from the original impulse model ${ }^{3}$ by the first term, which was previously assumed to equal unity. The reader is referred to previous literature for a discussion of the effect of the specific heat ratio $^{5,6} \gamma$ and the specific energy content ${ }^{3} q / R T_{1}$, whereas this study addresses the effect of $P_{0} / P_{1}$ on the impulse and the parameter $K$. The values of $K$ and $\beta$ are determined from experimental data.

Decreasing environmental pressure in Eq. (5) increases specific impulse (holding all else constant, including $K$ ), which is due to an increase in the pressure difference $P_{3}-P_{0}$. Changes in the environmental pressure also affect the first and third terms of $K$ (Eq. (6). The first term of $K$ varies with the environmental pressure only with variations in the $P_{0} / P_{1}$ parameter. The pressure ratio $P_{3} / P_{1}$ is known ${ }^{3}$ to depend only on the properties (specific heat ratio $\gamma$ and energy content $q / R T_{1}$ ) of the initial mixture. Thus, decreasing $P_{0} / P_{1}$ results in decreasing the first term of Eq. (6) for $P_{0} / P_{1}<1$. Decreasing $P_{0} / P_{1}$ causes an increase in the third term of Eq. (6) by increasing $\beta$. It is this increase in $\beta$ (representing the blowdown time) that causes measurable increases in $K$ and $I_{\mathrm{SP}}$ to occur. Thus, the specific impulse of a detonation tube depends on mixture properties $\left(\gamma\right.$ and $\left.q / R T_{1}\right)$ and environmental pressure $P_{0} / P_{1}$.

The similarities between factors contributing to the unsteady impulse equation above and the impulse from ideal steady-flow expansion should be noted. Consider a rocket operating at a given environmental pressure and assume the combustion products adiabatically expand within the nozzle. Here the energy content of the products is represented by the total enthalpy $h_{t}$, which remains constant throughout the nozzle. ${ }^{7,8}$ The nozzle area ratio, along with the continuity equation and $\gamma$ of the products, determines the degree of product expansion, represented by the ratio of pressure at the nozzle exhaust to total pressure $P_{t}$ in the combustion chamber. If the nozzle expansion ratio is optimized so that the exhaust pressure and the environmental pressure are equal, then the nozzle area ratio does not need to be known explicitly. Thus, the specific impulse of a steady-flow rocket engine (Eq. (7)) depends, as in the case of a detonation tube, on the mixture properties (specific heat ratio $\gamma$ and energy content $h_{t}$ ) and the environment pressure $P_{0} / P_{t}$ :

$$
I_{\mathrm{SP}}=(1 / g) \sqrt{h_{t}\left[1-\left(P_{0} / P_{t}\right)^{(\gamma-1) / \gamma}\right]}
$$

Although directly comparing unsteady impulse from a detonation tube to steady impulse from a rocket is not strictly valid, the fact that both depend on the product specific heat ratio, the energy content in the products, and the environmental pressure ratio implies that comparisons can be made, if this is done carefully. The value of $\gamma$ remains the same in both the steady and unsteady flow cases. A steady flow equivalent pressure ratio for the case of the detonation tube is needed to facilitate a meaningful comparison. This is discussed further in a later section.

\section{Experimental Facility}

The detonation tube had an inner diameter of $76.2 \mathrm{~mm}$ and a length of $1.014 \mathrm{~m}$. One end of the tube was closed, forming the thrust surface, whereas the other end was open. Mylar diaphragms with thicknesses of 25,51 , and $105 \mu \mathrm{m}$ initially sealed the open end, separating the combustible ethylene-oxygen mixture from the environmental air. Three pressure transducers and ten ionization gauges measured wave arrival times and pressure histories at specific axial locations. The mixture was ignited at the thrust surface by a standard aircraft spark plug with discharge energy $30 \mathrm{~mJ}$. Due to the low spark energy, detonations were obtained by transition from an initial deflagration.

The detonation tube was hung in a ballistic pendulum arrangement within a large tank (the tank was actually the test section and dump tank of the T5 hypersonic wind tunnel facility at Caltech) as illustrated in Fig. 4. The "environment" refers to the volume internal to the tank but external to the detonation tube and consisted of room air at pressure $P_{0}$ between 100 and $1.4 \mathrm{kPa}$. The initial pressure $P_{1}$ of the combustible mixture inside the detonation tube varied between 100 and $30 \mathrm{kPa}$.

The cylindrical tank had an inner diameter of $2 \mathrm{~m}$, a length of $4 \mathrm{~m}$, and an internal volume of approximately $12,500 \mathrm{~L}$. The attached test section (labeled in Fig. 4) is a cylinder approximately $0.7 \mathrm{~m}$ in diameter and $1.3 \mathrm{~m}$ in length. It incorporated two windows through which the tube motion was observed by means of a ruler extending from the thrust surface. Movement of the ruler was filmed by a digital camera situated outside the tank. The maximum deflection of the tube was converted into impulse (Eq. (8)) by applying the classical analysis of an impulsively created motion and the conservation of energy:

$$
I_{\mathrm{SP}}=\left(M_{p} / g \rho_{1} V\right) \sqrt{2 g L_{p}\left(1-\sqrt{1-\left(\Delta x / L_{p}\right)^{2}}\right)}
$$

This expression is exact and there are no limits on the values of $\Delta x$. Actual values of $\Delta x$ observed in our experiments were between 39 and $292 \mathrm{~mm}$. The experimental uncertainty in the specific impulse was estimated to be $\pm 3.8 \%$.

A feedthrough plate located on the bottom of the tank test section (Fig. 4) passed the gas lines and electrical connections through the tank wall to the detonation tube. The initial ethylene-oxygen mixture was created by the method of partial pressures in an external mixing vessel. Prior to each test, the tank door was opened and a new diaphragm was installed. The tank door was then sealed and the desired environmental pressure was established with a dedicated vacuum pump. The detonation tube was evacuated with a second vacuum pump to at least $133 \mathrm{~Pa}$ and then filled from the mixing vessel to the desired initial pressure.

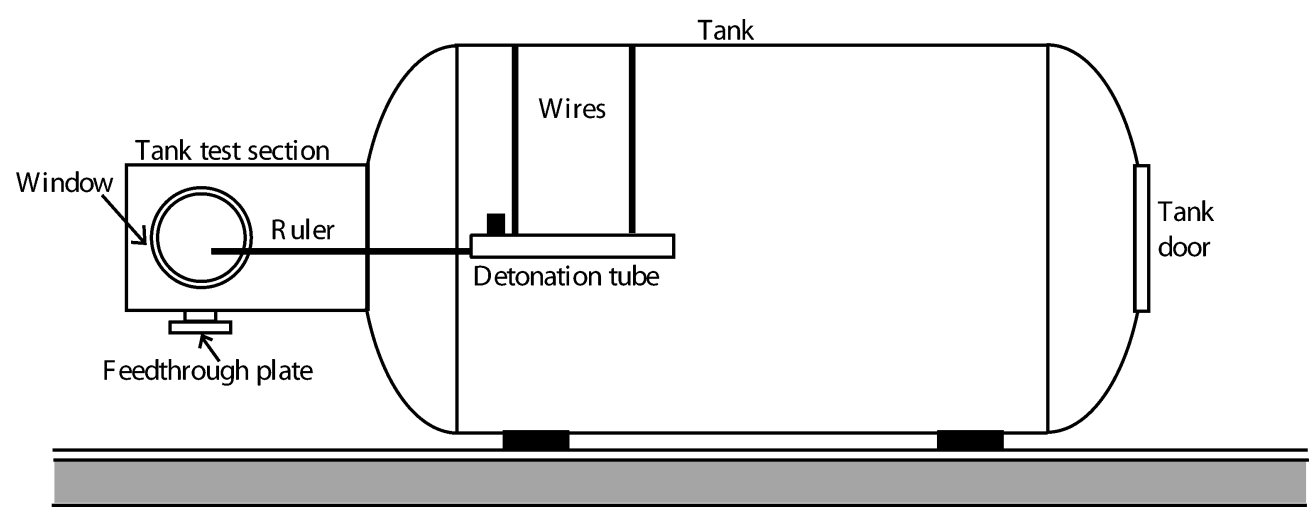

Fig. 4 Schematic of experimental facility. 
It should be noted that the exhaust of a practical PDE will not be sealed with a diaphragm. However, this experimental setup requires the use of a diaphragm to obtain repeatable single-cycle impulse data. The presence of the diaphragm has a nonnegligible effect on the measured impulse, as shown in the next section. The qualitative relationship between single-cycle impulse and environmental pressure sought in this work is not affected by the diaphragm.

\section{Experimental Data}

\section{A. Measured $\boldsymbol{U}_{\mathrm{CJ}}$ and $\boldsymbol{P}_{\mathbf{3}}$ Values}

Chapman-Jouguet detonation wave velocities of the ethyleneoxygen mixtures were measured from ionization gauge data and plateau pressures $P_{3}$ were measured from recorded pressure histories at each initial mixture pressure. Predicted values of ChapmanJouguet velocity and plateau pressure, average measured value, difference between the maximum and the minimum measured values, and standard deviation from the mean appear in Tables 1 and 2 respectively. ${ }^{9}$

Relative differences between the measured and predicted detonation velocities are less than $0.05 \%$ for mixtures with an initial pressure $60 \mathrm{kPa}$ or higher. Larger differences are observed for mixtures with lower initial pressures, but this is expected from the longer times required for transition to detonation. The relative difference between the measured and predicted plateau pressures is less than $4 \%$ for mixtures with an initial pressure of $60 \mathrm{kPa}$ or higher. The difference is less than $14 \%$ for mixtures with a lower initial pressure. Average measured values for the detonation velocity and plateau pressure were found to be independent of the environmental pressure.

\section{B. ISP Measured with 25- and 51- $\mu \mathrm{m}$ Diaphragms}

Impulse data obtained with 25 - and 51- $\mu$ m-thick diaphragms are plotted in Fig. 5 as a function of $P_{1}$. Data obtained at an environmental pressure of $100 \mathrm{kPa}$ agree with previous experimental data ${ }^{10} \mathrm{ob}-$ tained in a $50 \mathrm{~m}^{3}$ blastproof room within experimental uncertainty. Additional data at environment pressures of 54.5 and $16.5 \mathrm{kPa}$ are shown. The lines are polynomial curve fits to the data.

At an environmental pressure of $100 \mathrm{kPa}$, specific impulse decreases as initial mixture pressure decreases. This was noted previously and can be attributed to the increasing importance of dissociation with decreasing initial pressure. ${ }^{3,10}$ Because of the low ignition energy, recorded pressure histories illustrate the major deflagrationto-detonation (DDT) regimes previously ${ }^{10}$ documented. DDT was observed in these experiments for mixtures with initial pressures between 30 and $100 \mathrm{kPa}$. Since the purpose of this study was not to investigate DDT phenomena and experimental repeatability was poor under conditions of low $P_{0}$ and thin diaphragms, the remaining tests were carried out with values of $P_{1} \geq 60 \mathrm{kPa}$, where transition

Table 1 Measured $U_{\mathrm{CJ}}$ data for different initial pressures of stoichiometric ethylene-oxygen mixtures

\begin{tabular}{lcccc}
\hline \hline $\begin{array}{l}P_{1} \\
(\mathrm{kPa})\end{array}$ & $\begin{array}{c}U_{\mathrm{CJ}} \text { from } \\
\operatorname{Stanjan}^{9}(\mathrm{~m} / \mathrm{s})\end{array}$ & $\begin{array}{c}\text { Average } U_{\mathrm{CJ}} \\
\text { exps. }(\mathrm{m} / \mathrm{s})\end{array}$ & $\begin{array}{c}\text { Max-min } U_{\mathrm{CJ}} \\
\text { exps. }(\mathrm{m} / \mathrm{s})\end{array}$ & $\begin{array}{c}\text { Std. dev. of } U_{\mathrm{CJ}} \\
\text { exps. }(\mathrm{m} / \mathrm{s})\end{array}$ \\
\hline 100 & 2376 & 2375 & 63 & 24 \\
80 & 2365 & 2366 & 63 & 21 \\
60 & 2351 & 2350 & 90 & 32 \\
40 & 2331 & 2351 & 12 & 6 \\
30 & 2317 & 2352 & 221 & 93 \\
\hline
\end{tabular}

Table 2 Measured $\boldsymbol{P}_{3}$ data for different initial pressures of stoichiometric ethylene-oxygen mixtures

\begin{tabular}{lcccc}
\hline \hline $\begin{array}{l}P_{1} \\
(\mathrm{kPa})\end{array}$ & $\begin{array}{c}P_{3} \text { from } \\
\text { model }^{3}(\mathrm{MPa})\end{array}$ & $\begin{array}{c}\text { Average } P_{3} \\
\text { exps. (MPa) }\end{array}$ & $\begin{array}{c}\text { Max-min } P_{3} \\
\text { exps. (MPa) }\end{array}$ & $\begin{array}{c}\text { Std. dev. of } P_{3} \\
\text { exps. (MPa) }\end{array}$ \\
\hline 100 & 1.222 & 1.202 & 0.046 & 0.016 \\
80 & 0.970 & 0.982 & 0.035 & 0.012 \\
60 & 0.720 & 0.746 & 0.048 & 0.015 \\
40 & 0.472 & 0.523 & 0.009 & 0.004 \\
30 & 0.351 & 0.398 & 0.056 & 0.024 \\
\hline
\end{tabular}

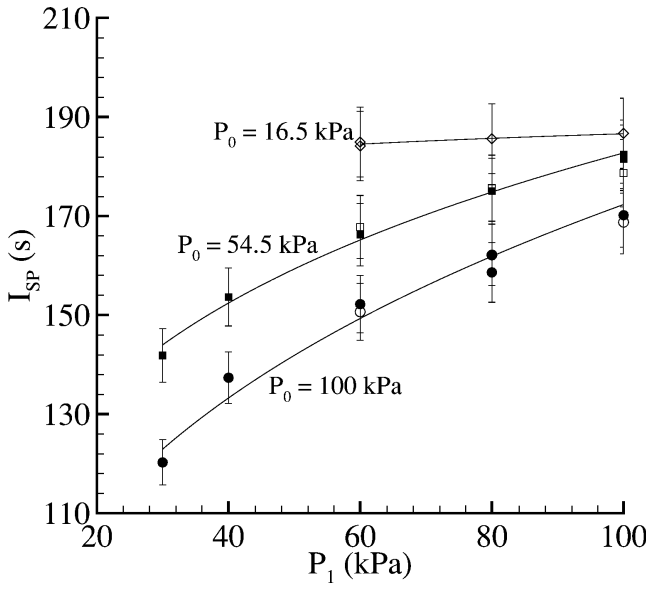

Fig. 5 Specific impulse data in tubes with a 25- (solid symbols)- or 51- $\mu \mathrm{m}$ (open symbols)-thick diaphragm. Initial mixture pressure varied between 100 and $30 \mathrm{kPa}$ and environmental pressure was 100, 54.5, or $16.5 \mathrm{kPa}$.

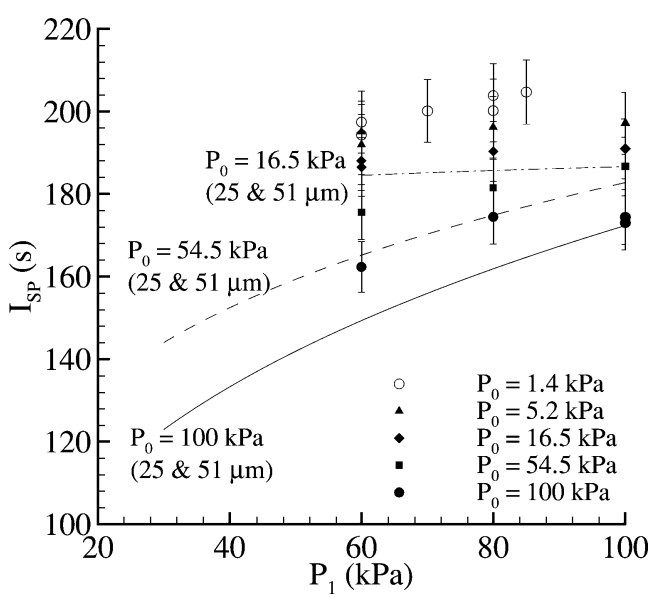

Fig. 6 Specific impulse data in tubes with a $105-\mu \mathrm{m}$ diaphragm as a function of initial mixture pressure. Data are plotted for environment pressures between $100 \mathrm{kPa}$ and $1.4 \mathrm{kPa}$.

to a detonation occurred within $4 \mathrm{~cm}$ of the thrust surface. At lower values of $P_{1}$, DDT occurs later in the tube after a period of flame acceleration and the leading compression waves cause the diaphragm to rupture, spilling some of the unburned mixture outside of the tube. This effect has been previously observed ${ }^{10}$ for initial pressures below $30 \mathrm{kPa}$, but here we observed this effect for initial pressures below $60 \mathrm{kPa}$ when the environmental pressure was low. In an effort to prevent early diaphragm rupture at low environmental pressures, a thicker diaphragm of $105 \mu \mathrm{m}$ was used.

\section{C. $I_{\text {SP }}$ Measured with 105- $\mu \mathrm{m}$ Diaphragms}

Impulse data obtained as a function of initial mixture pressure in tubes sealed with a 105- $\mu$ m-thick diaphragm appear in Fig. 6. The data at $P_{1}=100 \mathrm{kPa}$ with a $105-\mu \mathrm{m}$-thick diaphragm do not follow the same trend as shown in Fig. 5. This is due to the thicker diaphragm, which does not break quickly when the environmental pressure is near $100 \mathrm{kPa}$. The additional time required for the combustion wave to rupture the diaphragm results in energy loss due to heat transfer to the tube walls, affecting experimental repeatability. Evidence of diaphragm melting was observed after the experiments at $P_{0}=100 \mathrm{kPa}$ by examining the remaining diaphragm material that was not destroyed by the detonation wave. At the lower environmental pressures, evidence of diaphragm melting was not observed and repeat shots generated impulse values within the range of experimental uncertainty. 


\section{Analysis}

A. Determination of $\boldsymbol{\beta}$

Measured impulse values from Figs. 5 and 6 were used with Eq. (5) and the predicted values of $U_{\mathrm{CJ}}$ and $P_{3}$ from Tables 1 and 2 to determine $K$ as a function of $P_{0}$. The results are plotted in Fig. 7 along with $K=4.3$, used in the original impulse model. ${ }^{3}$ The scatter in the data can be attributed to the different diaphragm thicknesses.

A curve fit through the data of Fig. 7a yields a relationship (Eq. (9)) between $K$ and the pressure ratio $P_{0} / P_{1}$, which is plotted by the solid line. Alternatively, a relationship (Eq. (10)) between $K$ and the pressure ratio $P_{3} / P_{0}$ is shown by the solid line in Fig. 7b:

$$
\begin{gathered}
K=4.345\left(P_{0} / P_{1}\right)^{-0.023} \\
K=4.345\left[\left(P_{0} / P_{3}\right) \times\left(P_{3} / P_{1}\right)\right]^{0.023}
\end{gathered}
$$

Experimental values of $\beta$ are calculated with Eq. (6) using a constant value ${ }^{3}$ of $\alpha$ equal to 1.1 and the experimental values of $K$ (Fig. 7a). Similarly, an empirical relationship for $\beta$ as a function of $P_{0} / P_{1}$ is determined using the relationship for $K$ in Eq. (9) with Eq. (6). Both the individual values of $\beta$ and the continuous empirical relationship for $\beta$ appear in Fig. 8 as functions of $P_{0} / P_{1}$.

\section{B. Specific Impulse versus $\boldsymbol{P}_{\mathbf{0}}$}

Impulse data at initial pressures of 100, 80, and $60 \mathrm{kPa}$ (Figs. 5 and 6) are plotted in Figs. 9-11 as functions of the environmental pressure. For each initial pressure, the impulse increases as the environment pressure decreases. Also plotted are the predictions of Eq. (5) with $\beta=0.53$ from Wintenberger et al. ${ }^{3} \mathrm{~A}$ constant value of $\beta$ results in a linear increase in $I_{\mathrm{SP}}$ with decreasing $P_{0}$ for fixed values of $P_{3}$ and $P_{1}$. Experimental data best match the predictions

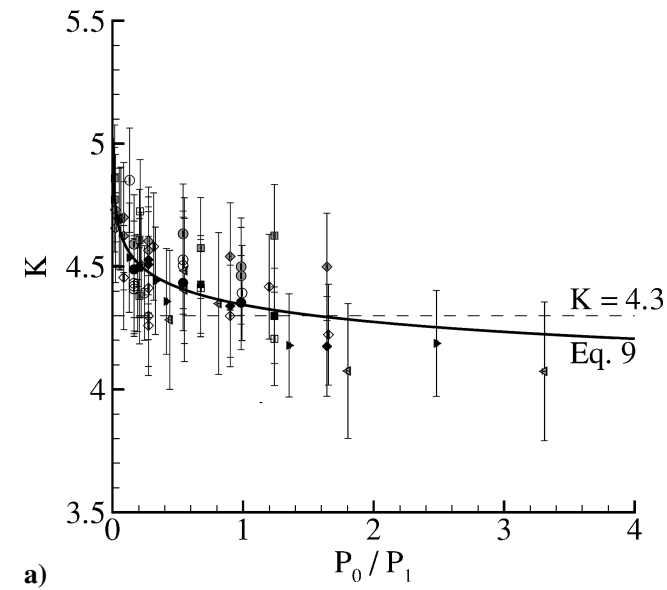

a)

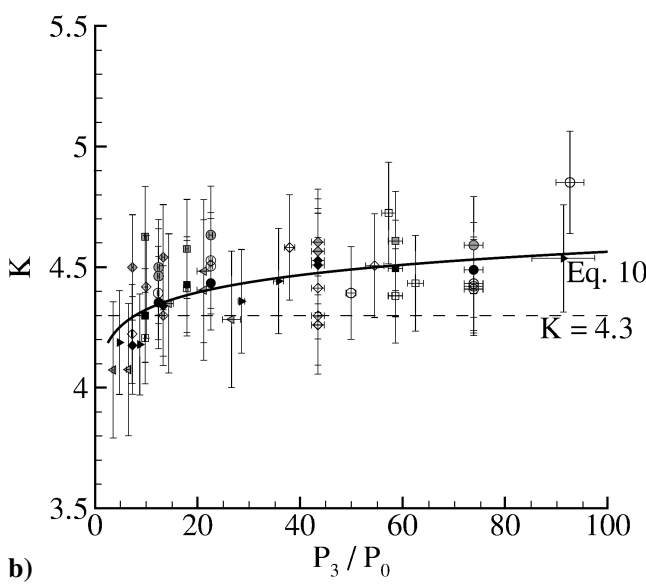

Fig. 7 Determination of $K$ as a function of a) $P_{0} / P_{1}$ and b) $P_{3} / P_{0}$ with error bars. Solid lines are the curve fit equations. Open symbols correspond to $25-\mu \mathrm{m}$ diaphragm, solid black symbols correspond to $51-\mu \mathrm{m}$ diaphragm, and solid gray symbols correspond to $105-\mu \mathrm{m}$ diaphragm.

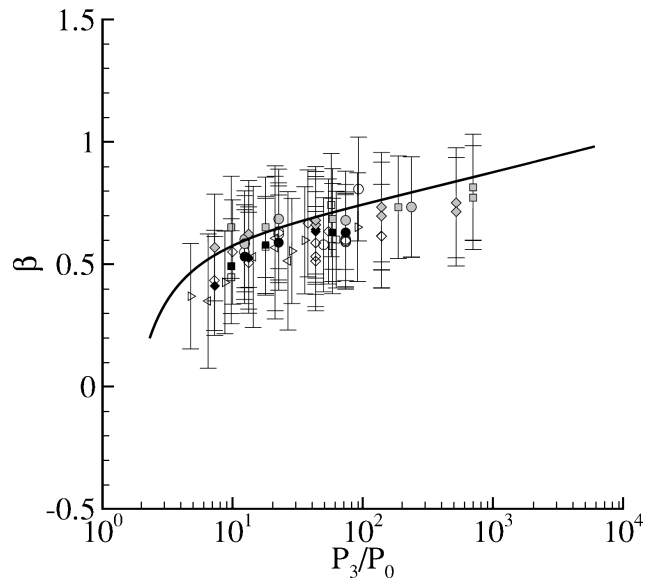

Fig. $8 \beta$ as a function of $P_{3} / P_{0}$. Open symbols correspond to $25-\mu \mathrm{m}$ diaphragm, solid black symbols correspond to 51- $\mu \mathrm{m}$ diaphragm, and solid gray symbols correspond to $105-\mu \mathrm{m}$ diaphragm.

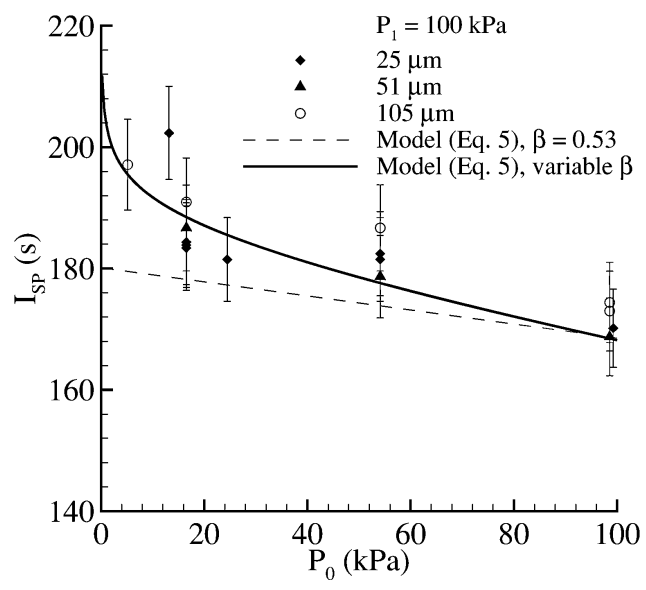

Fig. 9 Specific impulse data as a function of $P_{0}$ for an initial mixture pressure of $100 \mathrm{kPa}$.

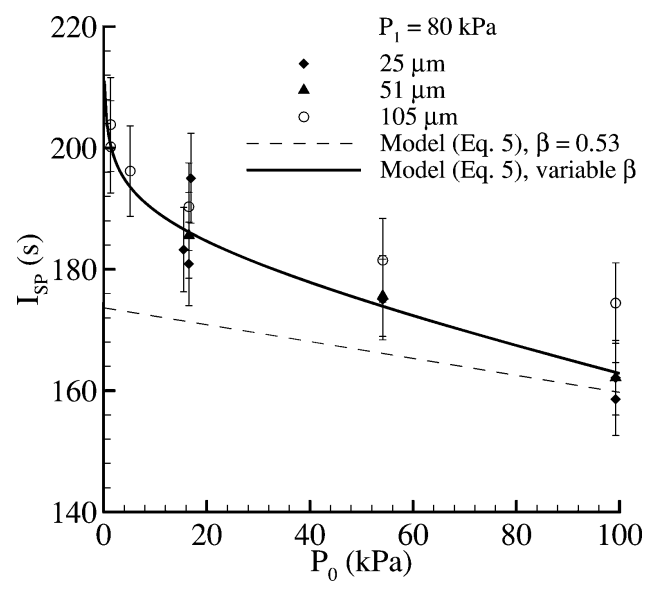

Fig. 10 Specific impulse data as a function of $\boldsymbol{P}_{\mathbf{0}}$ for an initial mixture pressure of $80 \mathrm{kPa}$.

of Eq. (5) with the constant value of $\beta$ when $P_{0}=P_{1}$ and the diaphragm is thin. This is expected because these are the conditions under which the parameters $K$ and $\beta$ of the original impulse model ${ }^{3}$ were derived. Experimental data clearly show an increase in the specific impulse greater than predicted if the blowdown time $t_{3}$ or equivalent $\beta$ remains constant. The experimental data are predicted if the value of $\beta$ is defined to be a function of the environmental pressure.

The data at initial pressure $80 \mathrm{kPa}$ are investigated further to determine the relative change in the parameters contributing to the 


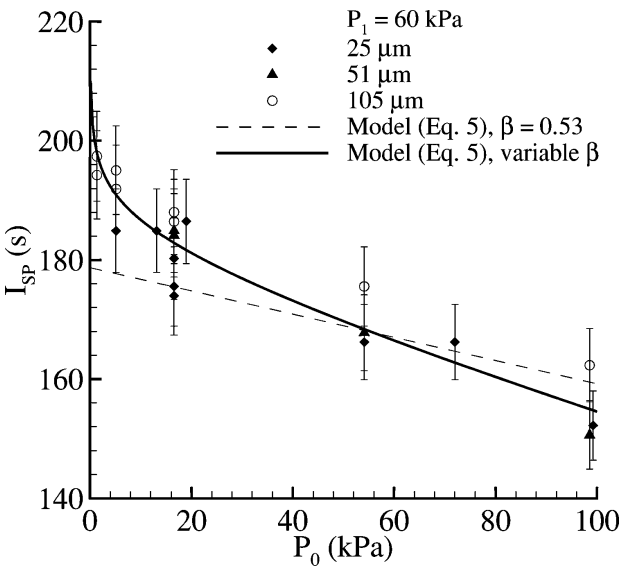

Fig. 11 Specific impulse data as a function of $\boldsymbol{P}_{0}$ for an initial mixture pressure of $60 \mathrm{kPa}$.

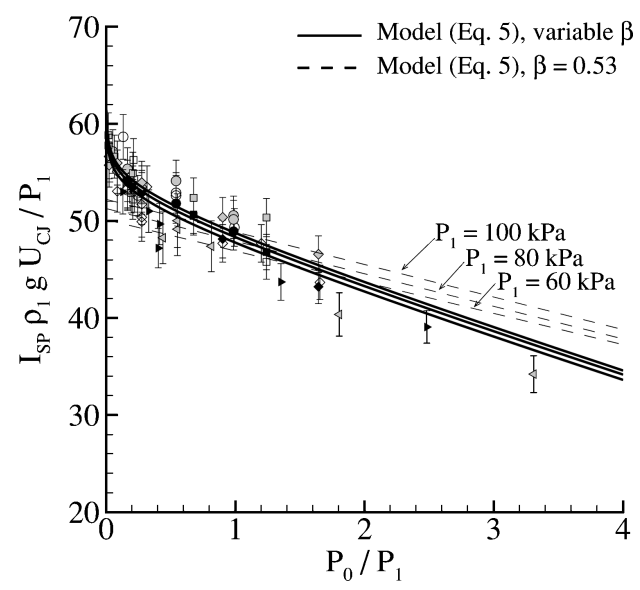

Fig. 12 Nondimensionalized impulse data plotted as a function of $P_{0} / P_{1}$. Data correspond to initial mixture pressures between 100 and $30 \mathrm{kPa}$, environmental pressures between 100 and $1.4 \mathrm{kPa}$, and diaphragm thickness of 25 (open symbols), 51 (solid black symbols), and $105 \mu \mathrm{m}$ (solid gray symbols).

measured impulse. Decreasing the environmental pressure from 100 to $1.4 \mathrm{kPa}$, a $99 \%$ decrease, results in the measured impulse increasing from 174 to $202 \mathrm{~s}$, a $16 \%$ increase. In the impulse equation (Eq. (5)), the only two parameters that change are $K$ and $P_{0} / P_{1}$. From Eq. (9), $K$ increases from 4.323 to 4.769 , a $10 \%$ increase, due to an increase in the first and third terms of Eq. (6).

\section{Nondimensionalized Impulse Data}

Nondimensionalization of the experimental data arises from a key relationship within the impulse model (Eq. (5)):

$$
I_{\mathrm{SP}} \rho_{1} g U_{\mathrm{CJ}} / P_{1}=K\left(P_{3} / P_{1}-P_{0} / P_{1}\right)
$$

The nondimensional group $I_{\mathrm{SP}} \rho_{1} g U_{\mathrm{CJ}} / P_{1}$ appears. The ratio $P_{3} / P_{1}$ is known ${ }^{3}$ to depend on $\gamma$ of the products and the energy content $q / R T_{1}$. Thus, the scaling of Eq. (11) results in Fig. 12, which is plotted as a function of the pressure ratio $P_{0} / P_{1}$. All data of Figs. 5 and 6 are shown in Fig. 12 and the scatter in the data is due to the different diaphragm thicknesses. The three lines in each series correspond to initial pressures of 100,80 , and $60 \mathrm{kPa}$.

Alternatively, the impulse can be written as

$$
I_{\mathrm{SP}} \rho_{1} g U_{\mathrm{CJ}} / P_{1}=K\left(P_{0} / P_{1}\right)\left[\left(P_{3} / P_{0}\right)-1\right]
$$

where the nondimensional group $I_{\mathrm{SP}} \rho_{1} g U_{\mathrm{CJ}} / P_{1}$ again appears along with an important pressure ratio, $P_{3} / P_{0}$. Figure 13 replots the data as a function of $P_{3} / P_{0}$, which better illustrates the effect of environmental pressure, because it is difficult to distinguish the individual data points at pressure ratios $P_{0} / P_{1}<0.5$ in Fig. 12 .

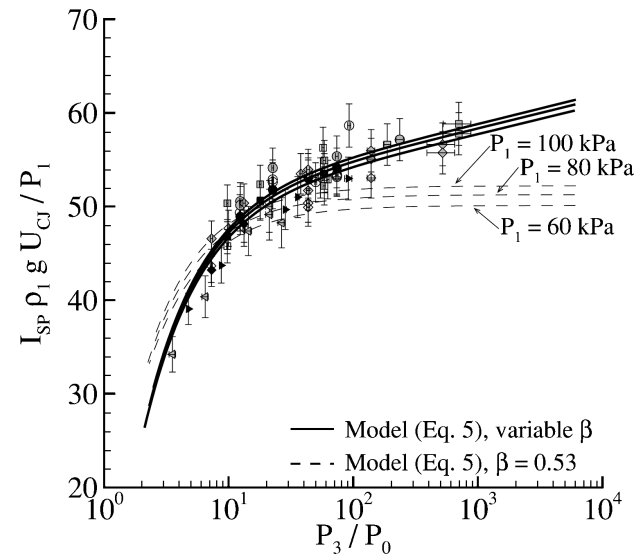

Fig. 13 Nondimensionalized impulse data plotted as a function of $P_{3} / P_{0}$. Data correspond to initial mixture pressures between 100 and $30 \mathrm{kPa}$, environmental pressures between 100 and $1.4 \mathrm{kPa}$, and diaphragm thickness of 25 (open symbols), 51 (solid black symbols), and $105-\mu \mathrm{m}$ (solid gray symbols).

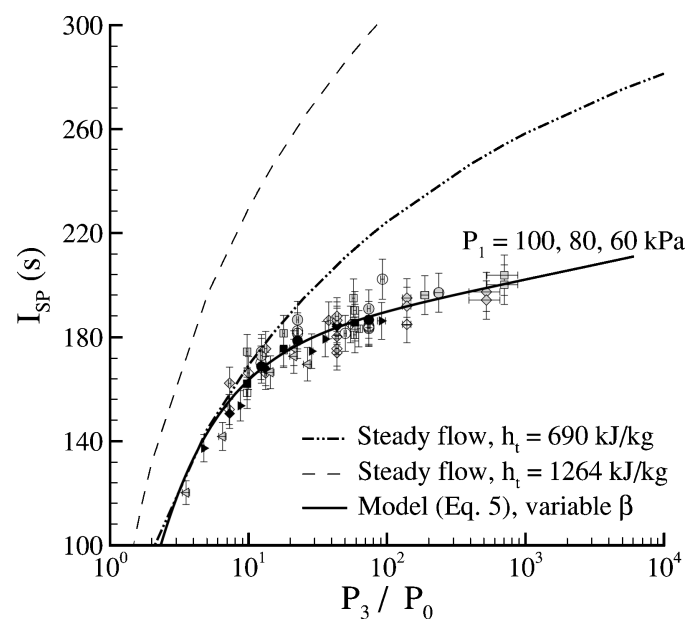

Fig. 14 Specific impulse data plotted as a function of $\boldsymbol{P}_{3} / \boldsymbol{P}_{0}$. Data correspond to initial mixture pressures between 100 and $30 \mathrm{kPa}$, environmental pressures between 100 and $1.4 \mathrm{kPa}$, and diaphragm thickness of 25 (open symbols), 51 (solid black symbols), and $105 \mu \mathrm{m}$ (solid gray symbols). Thin solid curves corresponds to ideal impulse from a steady flow nozzle for values of $h_{t}=1264$ and $690 \mathrm{~kJ} / \mathrm{kg}$. Thick solid curve corresponds to the model predictions with variable $\beta$.

Steady flow predictions for two values of $h_{t}$ are plotted with the experimental data in Fig. 14 as a function of $P_{3} / P_{0}$. Although direct comparison between the steady flow predictions and the detonation tube impulse is not strictly valid, as was discussed previously, the comparison can be used to evaluate the effect of exhaust gas underexpansion and the magnitude by which a perfectly designed nozzle may increase the impulse over the baseline case of a straight detonation tube. In evaluating the impulse of the steady flow case, a choice for the total enthalpy $h_{t}$ must be made that best represents the specific energy content $q / R T_{1}$ of the detonation tube. This choice is not straightforward due to the time dependency of the flow exiting the detonation tube.

The first obvious choice for $h_{t}$ is the condition in state 3 , the stagnant flow region behind the Taylor wave. State 3 is present at the thrust surface during a significant fraction of the cycle time $\left(t_{1}+t_{2}\right) /\left(t_{1}+t_{2}+t_{3}\right)$. A value of $h_{t}=1264 \mathrm{~kJ} / \mathrm{kg}$ is equivalent to state 3 in the detonation tube with an initial mixture of ethylene and oxygen at $P_{1}=80 \mathrm{kPa}$. In this case, the steady flow predictions overestimate the measured impulse data from the detonation tube even at low pressure ratios. This indicates that the chosen value of $h_{t}$ does not adequately represent the unsteady case.

A better choice for $h_{t}$ would be the average state in the detonation tube during a complete cycle (i.e., $T=\left(T_{3}+T_{0}\right) / 2$, 
$\left.P=\left(P_{3}+P_{0}\right) / 2\right)$. This is because the average pressure inside the detonation tube is lower than in state 3 because the products are exhausting from the tube during most of the cycle time. For $P_{1}=80 \mathrm{kPa}$, using the average pressure inside the detonation tube results in $h_{t}=690 \mathrm{~kJ} / \mathrm{kg}$. This is plotted in Fig. 14 and better represents the experimental data.

As the pressure ratio across the nozzle increases, the difference between the experimental data and the theoretical steady flow impulse increases indicating the lack of complete product gas expansion to the lower environment pressures. These experimental data of a detonation tube at different environment pressures serves as a baseline from which the effect of adding a nozzle can be quantified.

\section{Summary}

This study obtained the first experimental data quantifying the effect of environmental pressure on the single-cycle impulse of a fully filled detonation tube. Data were obtained for stoichiometric mixtures of ethylene and oxygen at initial pressures between 100 and $30 \mathrm{kPa}$ and environment pressures between 100 and $1.4 \mathrm{kPa}$. The specific impulse increased as the environment pressure decreased and the initial mixture pressure remained constant. This increase in impulse was not predicted by the original impulse model, ${ }^{3}$ which used a constant value of $K$ and $\beta$. At the lowest environment pressures, increased blowdown time caused the impulse to increase approximately $11 \%$ over the original impulse predictions. New model parameters $K=K\left(\gamma, q / R T_{1}, P_{0} / P_{1}\right)$ and $\beta=\beta\left(\gamma, q / R T_{1}, P_{0} / P_{1}\right)$ were determined from the experimental data and defined as functions of the environment pressure. Impulse predictions assuming full expansion from average conditions inside the detonation tube were compared to the impulse of a detonation tube, indicating that the detonation tube exhaust products are underexpanded.

\section{Acknowledgment}

This work was supported by the Office of Naval Research Multidisciplinary University Research Initiative Multidisciplinary Study of Pulse Detonation Engine (N00014-02-1-0589), and General Electric Contract GE-PO A02 81655 under DABT-63-0-0001.

\section{References}

${ }^{1}$ Wintenberger, E., "Application of Steady and Unsteady Detonation Waves to Propulsion," Ph.D. Dissertation, Dept. of Aeronautics, California Institute of Technology, June 2004.

${ }^{2} \mathrm{Wu}$, Y., Ma, F., and Yang, V., "System Performance and Thermodynamic Cycle Analysis of Air-Breathing Pulse Detonation Engines," Journal of Propulsion and Power, Vol. 19, No. 4, 2003, pp. 556-567.

${ }^{3}$ Wintenberger, E., Austin, J. M., Cooper, M., Jackson, S., and Shepherd, J. E., "Analytical Model for the Impulse of a Single-Cycle Pulse Detonation Tube," Journal of Propulsion and Power, Vol. 19, No. 1, 2003, pp. 22-38.

${ }^{4}$ Falempin, F., Bouchaud, D., Forrat, B., Desbordes, D., and Daniau, E., "Pulsed Detonation Engine Possible Application to Low Cost Tactical Missile and to Space Launcher," AIAA Paper 2001-3815, July 2001.

${ }^{5}$ Wintenberger, E., Austin, J. M., Cooper, M., Jackson, S., and Shepherd, J. E., "Reply to Comment on "Analytical Model for the Impulse of Single-Cycle Pulse Detonation Tube' (Wintenberger et al., Journal of Propulsion and Power, Vol. 19, No. 1, pp. 22-38, 2003), by W. H. Heiser and D. T. Pratt," Journal of Propulsion and Power, Vol. 20, No. 1, 2004, pp. 189-191.

${ }^{6}$ Wintenberger, E., Cooper, M., Pintgen, F., and Shepherd, J. E., "Reply to Comment on 'Analytical Model for the Impulse of Single-Cycle Pulse Detonation Tube' (Wintenberger et al., Journal of Propulsion and Power, Vol. 19, No. 1, pp. 22-38, 2003), by M. I. Radulescu and R. K. Hanson," Journal of Propulsion and Power, Vol. 20, No. 5, 2004, pp. 957-959.

${ }^{7}$ Sutton, G. P., Rocket Propulsion Elements, 6th ed., Wiley, New York, 1992, pp. 41-68.

${ }^{8}$ Hill, P. G., and Peterson, C. R., Mechanics and Thermodynamics of Propulsion, 2nd ed., Addison-Wesley, Reading, MA, 1992, pp. 513-540.

${ }^{9}$ Reynolds, W., "The Element Potential Method for Chemical Equilibrium Analysis: Implementation in the Interactive Program STANJAN," Technical Reports, Mechanical Engineering Department, Stanford University, 1986.

${ }^{10}$ Cooper, M., Jackson, S., Austin, J. M., Wintenberger, E., and Shepherd, J. E., "Direct Experimental Impulse Measurements for Detonations and Deflagrations," Journal of Propulsion and Power, Vol. 18, No. 5, 2002, pp. 1033-1041. 